\title{
PERSPECTIVE
}

\section{Chiral discrimination in NMR spectroscopy}

\section{A. David Buckingham*}

Department of Chemistry, Cambridge University, Cambridge CB2 1EW, UK

Quarterly Reviews of Biophysics (2015), 48(4), pages 421-423 doi:10.1017/S0033583515000074

Abstract. Nuclear magnetic resonance is the most important form of molecular spectroscopy in chemistry and biochemistry but it is normally blind to chirality. It was predicted in 2004 that precessing nuclear spins in chiral molecules in a liquid in a strong magnetic field induce a rotating electric polarization that is of opposite sign for enantiomers. This polarization arises from the distortion of the electronic structure by the nuclear magnetic moment in the presence of the strong magnetic field and is equivalent to the linear effect of an electric field on the nuclear shielding tensor. The polarization is strongly enhanced in dipolar molecules through the partial orientation of the permanent dipole through the antisymmetric part of the nuclear magnetic shielding tensor. Alternatively, an applied electric field will induce a chirally sensitive magnetization perpendicular to the field and to the nuclear spin. Progress towards the experimental realization of chiral discrimination by NMR is assessed.

Key words: Chiral discrimination NMR spectroscopy Pseudoscalar molecular properties.

\section{Introduction}

High-resolution nuclear magnetic resonance spectroscopy is an important technique for determining the structure of molecules in solution. The molecular properties that determine an NMR spectrum, namely the chemical shift and the nuclear spin-spin coupling constant, are identical for a molecule and its mirror image. These second-rank tensor properties $\sigma_{\alpha \beta}^{(\mathrm{N})}$ and $J_{\alpha \beta}^{\left(\mathrm{NN}^{\prime}\right)}$, where $\mathrm{N}$ and $\mathrm{N}^{\prime}$ are two nuclei with spin, are even under the parity operator $\hat{P}$ that inverts the coordinates of all particles in the molecule and therefore converts one enantiomer of a chiral molecule into the other. For chiral sensitivity one looks for properties that are even under time-reversal $\hat{T}$ and odd under $\hat{P}$, like the optical rotational strength, which is the imaginary part of the scalar product of electric and magnetic transition dipole moments (Barron, 2004). The linear dependence of $\sigma_{\alpha \beta}^{(\mathrm{N})}$ on an electric field $\boldsymbol{E}$ is determined by the third-rank tensor $\sigma_{\alpha \beta \gamma}^{(1)(\mathrm{N})}$ which is odd under $\hat{P}$ and even under $\hat{T}$. It has been shown (Buckingham, 2004; Buckingham \& Fischer, 2006) that the isotropic part of $\sigma_{\alpha \beta \gamma}^{(1)(\mathrm{N})}$, viz. $\bar{\sigma}^{(1)(\mathrm{N})} \varepsilon_{\alpha \beta \gamma}$, where $\varepsilon_{\alpha \beta \gamma}$ is the third-rank

\footnotetext{
* Author for correspondence: A. David Buckingham, Department of Chemistry, Cambridge University, Cambridge CB2 1EW, UK. Tel.: +44-1223-336458; Fax: +44-1223-336362; Email: adb1000@cam.ac.uk
}

skew-symmetric unit tensor and $\bar{\sigma}^{(1)(\mathrm{N})}=(1 / 6) \varepsilon_{\alpha \beta \gamma} \sigma_{\alpha \beta \gamma}^{(1)(\mathrm{N})}$ is a pseudoscalar which vanishes for achiral molecules, leads to a rotating electric polarization perpendicular to the precessing nuclear magnetic moment and to the applied magnetic field $\boldsymbol{B}^{(0)}$. Each line in the NMR spectrum of a chiral molecule has an associated positive or negative signal at the same frequency, as occurs in circular dichroism and vibrational circular dichroism (Stephens et al. 2012) but these chirally sensitive spectral lines are yet to be observed in NMR.

Computations of $\bar{\sigma}^{(1)(\mathrm{N})}$ (Buckingham \& Fischer, 2006; Lazzeretti et al. 2008; Monaco \& Zanasi 2011; Pelloni et al. 2013; Zanasi et al. 2007) have shown that its contribution to the electric polarization is likely to be too small for easy detection, even for heavier nuclei such as ${ }^{13} \mathrm{C}$ and ${ }^{19} \mathrm{~F}$. However, a recent Communication (Buckingham, 2014) showed that there is an additional contribution to the rotating polarization in the case of dipolar molecules; the permanent electric dipole moment $\mu^{(0)}$ contributes a temperature-dependent orientation polarization through the torque exerted on the dipole by the precessing nuclear magnetic moment through the antisymmetric part of $\sigma_{\alpha \beta}^{(\mathrm{N})}$. The molecular properties that give the rotating electric polarization associated with precessing nuclear spins 
following a $\pi / 2$ pulse are $\bar{\sigma}^{(1)(\mathrm{N})}+\bar{P}^{(\mathrm{N})} / k T$, where $k$ is the Boltzmann constant, $T$ the absolute temperature, and the pseudoscalar $\bar{P}^{(\mathrm{N})}$ for nucleus $\mathrm{N}$ is (a repeated Greek suffix implies a sum over all three Cartesian components)

$$
\begin{aligned}
\bar{P}^{(\mathrm{N})} & =\frac{1}{6}\left(\varepsilon_{\alpha \beta \gamma} \mu_{\alpha}^{(0)} \sigma_{\beta \gamma}^{(\mathrm{N})}\right) \\
= & \frac{1}{6}\left[\mu_{z}^{(0)}\left(\sigma_{x y}^{(\mathrm{N})}-\sigma_{y x}^{(\mathrm{N})}\right)+\mu_{x}^{(0)}\left(\sigma_{y z}^{(\mathrm{N})}-\sigma_{z y}^{(\mathrm{N})}\right)\right. \\
& \left.+\mu_{y}^{(0)}\left(\sigma_{z x}^{(\mathrm{N})}-\sigma_{x z}^{(\mathrm{N})}\right)\right]
\end{aligned}
$$

Computations on the HOOF molecule (Buckingham, 2014) indicate that at room temperature this orientation polarization is two or three orders of magnitude larger than the distortion polarization due to $\bar{\sigma}^{(1)}$, thus improving the prospects of achieving chiral discrimination in NMR spectroscopy.

A recent paper (Buckingham et al. 2015) reports results of computations of $\bar{\sigma}^{(1)}$ and $\bar{P} / k T$ for nine small chiral molecules containing heavier atoms such as ${ }^{13} \mathrm{C},{ }^{15} \mathrm{~N},{ }^{17} \mathrm{O},{ }^{19} \mathrm{~F}$, ${ }^{33} \mathrm{~S},{ }^{77} \mathrm{Se}$ and ${ }^{125} \mathrm{Te}$ as well as protons; it confirms the large enhancement due to dipolar orientation.

An alternative approach (Buckingham \& Fischer, 2006) to the detection of chirally sensitive NMR signals is through the precessing magnetization induced by an applied electric field $\boldsymbol{E}$. In an isotropic sample, this magnetization is proportional to the vector product of the nuclear magnetization and $\boldsymbol{E}$. Like the induced electric polarization, it is dependent on $\bar{\sigma}^{(1)}+\bar{P} / k T$, but the temperature-dependent term now arises from the orientation of the permanent dipole by the electric field; if $\boldsymbol{E}$ is a low-frequency field, then even large molecules will be able to respond to its oscillations. In the case of the precessing electric polarization arising from the torque exerted on the molecules through the antisymmetric part of the nuclear magnetic shielding tensor, the molecules need to be able to respond to the precessing nuclear magnetization, i.e. to the NMR frequency. This would be a problem for very large molecules.

\section{Discussion}

Chiral discrimination in high-resolution NMR spectroscopy could be observed through the rotating electric polarization generated by magnetic nuclei precessing in the plane perpendicular to the magnetic field $B^{(0)}$ following a $\pi / 2$ pulse (Buckingham, 2004; Buckingham \& Fischer, 2006) or through the magnetization induced by an electric field $\boldsymbol{E}$ (Buckingham \& Fischer, 2006). In both cases, the signal is determined by $\left|\bar{\sigma}^{(1)}+\bar{P} / k T\right|$ (Buckingham, 2014; Buckingham \& Fischer, 2006). For dipolar molecules and that includes all chiral molecules except those of $D_{n}$ symmetry - computations show that at room temperature the magnitude of $\bar{P} / k T$ is normally much greater than that of $\bar{\sigma}^{(1)}$ (Buckingham, 2014; Buckingham et al. 2015).
The nature of the torque orienting the molecule through the antisymmetric components of $\sigma_{\alpha \beta}^{(\mathrm{N})}$ can be understood as follows: the antisymmetric part of $\sigma_{\alpha \beta}^{(\mathrm{N})}$ can be represented as $\varepsilon_{\alpha \beta \gamma} \sigma_{\gamma}^{*(\mathrm{~N})}$ where $\sigma^{*(\mathrm{~N})}$ is a polar vector (Buckingham, 2014; Jeffreys, 1931). Thus for a nucleus $\mathrm{N}$ on the symmetry axis of a chiral molecule of $C_{3}$ symmetry, there is one non-zero component of $\sigma^{*(\mathrm{~N})}$ (Birss, 1966), namely $\sigma_{z}^{*(\mathrm{~N})}=\left(\sigma_{x y}^{(\mathrm{N})}-\sigma_{y x}^{(\mathrm{N})}\right) / 2$ where $x, y$ and $z$ are molecule-fixed axes. The antisymmetric shielding contribution to the nuclear shielding spin Hamiltonian $\sigma_{\alpha \beta}^{(\mathrm{N})} \mathrm{m}_{\alpha}^{(\mathrm{N})} B_{\beta}^{(0)}$ is $\varepsilon_{\alpha \beta \gamma} \sigma_{\gamma}^{*(\mathrm{~N})} \mathrm{m}_{\alpha}^{(\mathrm{N})} B_{\beta}^{(0)}=\sigma^{*(\mathrm{~N})} \cdot\left(\mathbf{m}^{(\mathrm{N})} \times \boldsymbol{B}^{(0)}\right)$; if $\sigma_{z}^{*(\mathrm{~N})}$ is negative there is a lowering of energy when the molecular $z$-axis is in the direction of the vector product of the nuclear magnetic moment $\mathbf{m}^{(\mathrm{N})}$ and the magnetic field $\boldsymbol{B}^{(0)}$, causing the molecule, with its dipole $\mu_{z}^{(0)}$, to favour this direction and leading to electric polarization precessing in the plane perpendicular to $\boldsymbol{B}^{(0)}$ at right angles to the nuclear magnetization.

In the case of the nuclear magnetization induced by an applied electric field $\boldsymbol{E}$, the energy of the molecule is changed by $-\boldsymbol{\mu}^{(0)} \cdot \boldsymbol{E}$, causing the antisymmetric part of the nuclear shielding tensor to induce magnetization in the direction $\mathbf{m}^{(\mathrm{N})} \times \boldsymbol{E}$ (Buckingham \& Fischer, 2006).

The results of computations of $\bar{\sigma}^{(1)}$ and $\bar{P} / k T$ (Buckingham, 2014; Buckingham et al. 2015) give hope that NMR experiments will soon provide chiral discrimination as in other forms of spectroscopy. Estimates of alternating voltages of the order of $1 \mathrm{nV}$ for signals from protons in pure chiral liquids (Buckingham \& Fischer, 2006) increase to $1 \mu \mathrm{V}$ and substantially higher for heavier nuclei because of the dominance of $\bar{P} / k T$. The contribution of the rotating dipole depends on the polar molecule being able to follow the precessing nuclear spins, but this should not be a problem for small polar molecules for which dielectric loss occurs at frequencies from 3 to $300 \mathrm{GHz}$ (Hill et al. 1969) but will be a factor for large molecules. Application of a lowfrequency electric field $\boldsymbol{E}$ and detection of the induced magnetization could obviate this problem as well as providing potentially valuable discrimination through the modulation of $\boldsymbol{E}$.

\section{Acknowledgements}

The author wishes to express his indebtedness to Professor Dr Peer Fischer, Dr Piotr Garbacz, Dr Steffen Krämer and Dr Geert Rikken for their involvement with him in the experimental search for chirally sensitive NMR signals.

\section{References}

Barron, L. D. (2004). Molecular Light Scattering and Optical Activity. Cambridge: Cambridge University Press, p. 33 and 270. 
BIRss, R. R. (1966). Symmetry and Magnetism. Amsterdam: NorthHolland Publishing Company.

Buckingham, A. D. (2004). Chirality in NMR spectroscopy. Chemical Physics Letters 398, 1-5.

Buckingham, A. D. (2014). Communication: permanent dipoles contribute to electric polarization in chiral NMR spectra. Journal of Chemical Physics 140, 011103-1-3.

Buckingham, A. D. \& Fischer, P. (2006). Direct chiral discrimination in NMR spectroscopy. Chemical Physics 324, 111-116.

Buckingham, A. D., Lazzeretti, P. \& Pelloni, S. (2015). Chiral discrimination in NMR spectroscopy: computation of the relevant molecular pseudoscalars. Molecular Physics (in press).

Hill, N. E., Vaughan, W. E., Price, A. H. \& Davies, M. (1969). Dielectric Properties and Molecular Behaviour. London: Van Nostrand Reinhold Company, p. 303.

Jefrreys, H. (1931). Cartesian Tensors. Cambridge: Cambridge University Press, chapter VII.
Lazzeretti, P., Soncini, A. \& Zanasi, R. (2008). Response tensors for chiral discrimination in NMR spectroscopy. Theoretical Chemistry Accounts 119, 99-106.

Monaco, G. \& Zanasi, R. (2011). Searching for a good candidate to perform a chiral nuclear magnetic resonance experiment in disordered phase: a study of 8,9-difluoro- $P$-hexahelicene. Chirality 23, 752-755.

Pelloni, S., Faglioni, F. \& Lazzeretti, P. (2013). Chiral discrimination via nuclear magnetic resonsnce spectroscopy. Rendiconti Fiz. Acc. Lincei 24, 283-289.

Stephens, P. J., Devlin, F. J. \& Cheeseman, J. R. (2012). VCD Spectroscopy for Organic Chemists. London: CRC Press.

Zanasi, R., Pelloni, S. \& Lazzeretti, P. (2007). Chiral discrimination via nuclear magnetic shielding polarizabilities from NMR spectroscopy: theoretical study of $\left(R_{\mathrm{a}}\right)$-1,3-dimethylallene, (2R)-2-methyloxirane, and (2R)- $N$-methyloxaziridine. Journal of Computational Chemistry 28, 2159-2163. 